lipoperoxidation inhibition and antitumor potential, may have contributed to their exceptional longevity.

E-mail address: alda_pereira@hotmail.com

http://dx.doi.org/10.1016/j.freeradbiomed.2018.04.536

\title{
The teas of Portuguese centenarians
}

A. Pereira Silva ${ }^{1,2,3}$, M Ceu Costa $^{4,5}$, Patrícia Rijo
J.P. Gorjão-Clara
7,8

${ }^{1}$ Alameda Primary Care Health Center, Ministry of Health, Lisbon, Portugal

${ }^{2}$ Genetics Laboratory, Environmental Health Institute - ISAMB, Faculty of Medicine, University of Lisbon, Lisbon, Portugal

${ }^{3}$ Instituto de Investigação Científica Bento da Rocha Cabral, Lisbon, Portugal

${ }^{4}$ CBIOS- Centro de Biociências/The Biosciences Research Center, Portugal

${ }^{5}$ ERISA- High School of Health Ribeiro Sanches, Portugal

${ }^{6}$ Phytochemistry and Food Sciences-Group Coordinator - CBIOS, Portugal

${ }^{7}$ Geriatric Universitary Unit of Faculty of Medicine, University of Lisbon, Portugal

${ }^{8}$ Academic Medical Center of Lisbon - North of Lisbon Hospital Center, Portugal

Introduction: Medicinal plants traditional consumption habits, can contribute to longevity.

Methods: A sample of 253 centenarian individuals in Portugal, both sexes, median age100 years, was studied, to verify past habits in relation to medicinal-interest plants use. It was compared with a control group median age 67 year, with a reduced theoretical probability of reaching 100 years.

Results: Among the 8 most cited plants, in decreasing frequency order, in centenarian's group: Lemon-balm, barley, lemon-verbena, orange (leaf-flower), linden, whig-plant, pennyroyal and mount-carqueja (Pterospartum-tridentatum); in the control group: Lemon-balm, lemonverbena, chamomile, linden, prince-herb, green-tea, lemon-tea and minttea. Whereas $28 \%$ of the control subjects reported not using infusion plants, in the centennial group, only $9.1 \%$ reported not routinely use them $(\chi 2=30,42, p<0.001)$. Among the 8 plants most marked by the centenarians that were not mentioned by the controls, they include barley, whig-plant, pennyroyal and mount-carqueja.

Conclusion: the high antioxidant power associated with the use of plants by centenarian individuals, determined by anti-free radical's activity, 